Nonlinear Processes in Geophysics (2001) 8: 265-279

Nonlinear Processes

in Geophysics

(c) European Geophysical Society 2001

\title{
A theory of the Earth's magnetic field and of sunspots, based on a self-excited dynamo incorporating the Hall effect
}

\author{
A. de Paor \\ Department of Electronic and Electrical Engineering, National University of Ireland, Dublin, Belfield, Dublin 4, Ireland
}

Received: 27 March 1999 - Revised: 10 September 1999 - Accepted: 30 November 2000

\begin{abstract}
A new viewpoint on the generation and maintenance of the Earth's magnetic field is put forward, which integrates self-exciting dynamo theory with the possibility of energy coupling along orthogonal axes provided by the Hall effect. A nonlinear third-order system is derived, with a fourth equation serving as an observer of unspecified geophysical processes which could result in field reversal. Lyapunov analysis proves that chaos is not intrinsic to this system. Relative constancy of one of the variables produces pseudo equilibrium in a second order subsystem and allows for self-excitation of the geomagnetic field. Electromagnetic analysis yields expressions for key parameters. Models for secular variations recorded at London, Palermo and at the Cape of Good Hope over the past four hundred years are offered. Offset of the Earth's magnetic axis from the geographic axis is central to time-varying declination, but its causes have not yet been established. Applicability of the model to the explanation of sunspot activity is outlined. A corroborating experiment published by Peter Barlow in 1831 is appended.
\end{abstract}

\section{Introduction}

A self-consistent theory of the Earth's magnetic field has been sought for several hundred years. In the early years of the 20th century, Larmor (1919) made the profound suggestion that the key might lie in self-excited dynamo action in fluids in relative rotation within the core. This has inspired a great wealth of theory and experiment related to the geodynamo, and, in particular, to the study of self-exciting Faraday-disk homopolar dynamos as low-dimensional models of complicated magnetohydrodynamic processes (Hide, 1997; Hide et al., 1998; Moroz et al., 1998). The possible role of chaos in geomagnetic field reversals has been allowed for in some of these models, but a caution has been enunciated by Turcotte (1992) in a study of chaos in the Rikitake

Correspondence to: A. de Paor (annraoi.depaor@ucd.ie) dynamo (1958) as a possible model for reversals in the geomagnetic field, and of chaos in the atmosphere via the famous Lorenz (1963) equations:

"It should be emphasised, however, that there is a fundamental difference between the Lorenz equations and the Rikitake dynamo equations. The Lorenz equations are derived directly from the appropriate equations for thermal convection. The Rikitake dynamo equations are rigorously applicable to the model dynamo but the relation of the model dynamo to the dynamics of the core is completely ad hoc."

The Rikitake dynamo suffers in any case from a physical flaw (Hide, 1997) and it is not at all certain that chaos, if it is truly manifested here, should be sought in the dynamics of the geodynamo.

In recent work on networks of self-excited dynamos driving various types of motor, Hide (1997) made the following remarks:

"Self-exciting Faraday-disk homopolar dynamos ... are of much interest in their own right as nonlinear electro-mechanical systems which exhibit rich behaviour, even in the simplest cases investigated. But it is not, of course, possible to relate disk-dynamo systems to MHD counterparts such as the geodynamo in any direct quantitative way. Qualitatively, however, the motors can be regarded as being roughly equivalent to eddies in the fluid accelerated by Lorentz forces. Such forces also couple the geodynamo operating in the Earth's liquid metallic outer core to the solid metallic inner core, which is thereby driven into relative motion ... like a motor."

The author was introduced to geodynamo theory by Professor Raymond Hide in 1998, in connection with the problem of proving the absence of chaos under certain conditions in a configuration studied by him (Hide, 1998; de Paor, 1998a). Since then, as time permitted, he has pondered why disk-dynamo systems have not been successfully related to their MHD counterparts. It occurred to him that the reason might lie in the circumstance that they do not exploit the potential which lies in the Lorentz equation for force on a charge moving in a magnetic field to act as an energy trans- 
fer mechanism between orthogonal axes.

The Lorentz force is manifested macroscopically as the Hall effect (Shockley, 1950), which is presented to undergraduate engineers as a tool for measuring electron and hole mobilities. Yet, as will be demonstrated below, it forms the basis for an energy coupler between orthogonal axes. That realisation proved to be the crucial link in the theory presented here.

The Hall effect was discussed briefly in a review of theories of the Earth's magnetism many years ago (Inglis, 1955). However, it was considered there as a possible adjunct to the thermoelectric effect, and was not integrated into any coherent model. The viewpoint adopted here is that it is a vital link in the chain of cause and effect, initiating and sustaining self-excitation in the geodynamo.

The theory to be explored here is that the metallic solid inner core of the Earth is rotating with respect to the mantle, as has been confirmed by Song and Richards (1996) and Vidale et al. (2000). This rotation maintains an angular velocity gradient across the outer core. The solid core is encircled by current in the right-hand screw direction, as viewed from the north, through the fluid core. This current generates the main geomagnetic field, which, due to the relative rotation, generates motional electromotive force in the fluid, driving current across the annulus, orthogonal to the magnetising current. The geomagnetic field passes down through the annulus and, again, via the Hall effect, feeds energy from the dynamo into the annulus. If conditions are right, positive feedback sustains the excitation in the face of Ohmic energy losses. The energy for this process must come from relative rotation of core and mantle, though what forces sustain that are unspecified here. As shown by Lyapunov analysis, if the system were autonomous, the null state would have global asymptotic stability. There is, hence, no intrinsic possibility of chaos, and any which is observed must come from forcing functions. Observations made by Hide (1998) are apt:

" In the interpretation of geomagnetic polarity reversals with their highly variable frequency over geological time it is necessary, as with other irregularly-fluctuating geophysical phenomena, to consider the relative importance of forced contributions associated with changing boundary conditions and of free contributions characteristic of the behaviour of nonlinear systems operating under fixed boundary conditions. New evidence - albeit indirect - in favour of the likely predominance of forced contributions is provided by the discovery reported here of the possibility of complete quenching of current fluctuations in a self-exciting homopolar dynamo driven by a steady couple applied to its single Faraday disk."

It is felt that a reasonable case is made in this paper that forced as opposed to free contributions are indeed responsible for any major fluctuations in the Earth's magnetic field.

\section{The Hall effect as an energy transfer mechanism}

The Hall effect is part of the stock-in-trade of every electrical engineer and physicist and yet, the author has never seen a

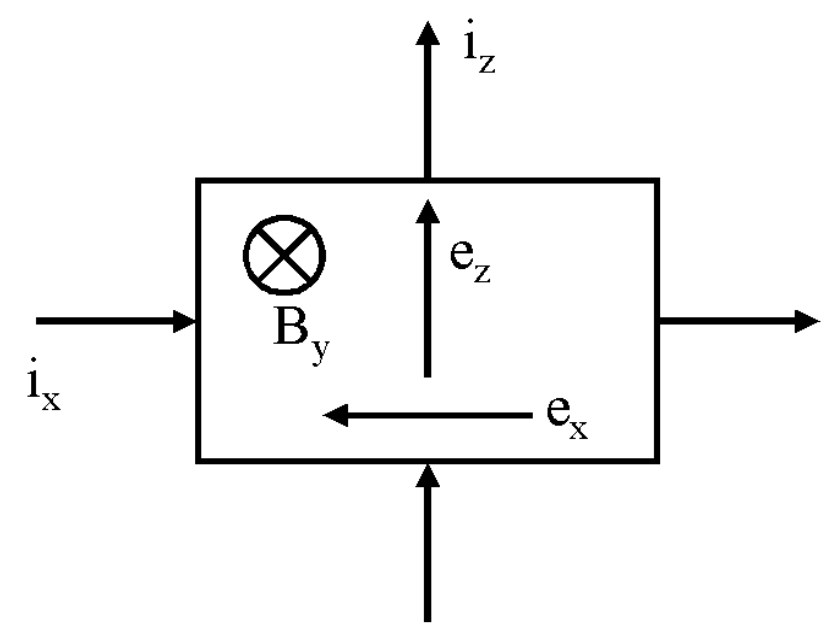

Fig. 1. Hall effect orthogonal axis energy transfer.

discussion of its role in energy transfer. It seems helpful, therefore, to set the scene by a brief treatment of this topic. We consider the situation depicted in Fig. 1. A sample, of length $l$ along the $x$ axis, breadth $b$ along the $z$ axis and depth $d$ along the $y$ axis, lies in a magnetic field of flux density $B_{y}$ directed downwards into the paper. A current $i_{x}$ flows to the right. A voltage $e_{z}$ is established, as shown, where

$e_{z}=R_{h} i_{x} \frac{B_{y}}{d}$

$R_{h}$ is the Hall coefficient of the material in question. Assuming conduction by both positive and negative carriers, having charges of equal magnitude $q$, in concentrations $p$ and $n$, respectively, and having mobilities $\mu_{p}$ and $\mu_{n}$, the following expression applies, subject to certain quantum-mechanical considerations (Shockley, 1950):

$R_{h}=\frac{p \mu_{p}^{2}-n \mu_{n}^{2}}{q\left(n \mu_{n}+p \mu_{p}\right)^{2}}$

$R_{h}$ may be positive or negative, or indeed, in a singular circumstance, zero. It is positive for $p$-type conduction and negative for $n$-type.

We now assume that the piece of material in Fig. 1 is allowed to drive current in the $z$ direction. This will generate a Hall voltage along the $x$ axis, governed by the same directional relationships as $e_{z}$. For $R_{h}$ positive, as was assumed in drawing $e_{z}$ in Fig. $1, e_{x}$ is in the negative $x$ direction and is given by the expression

$e_{x}=\frac{R_{h} i_{z} B_{y}}{d}$

The power fed into the material along the $x$ direction is given by $i_{x} e_{x}$ and extracted along the $z$ direction by $i_{z} e_{z}$. These two quantities are equal, and so the Hall effect provides a perfect orthogonal axis energy transfer mechanism, taking energy in along the $x$-axis and feeding it out along the $z$-axis. 


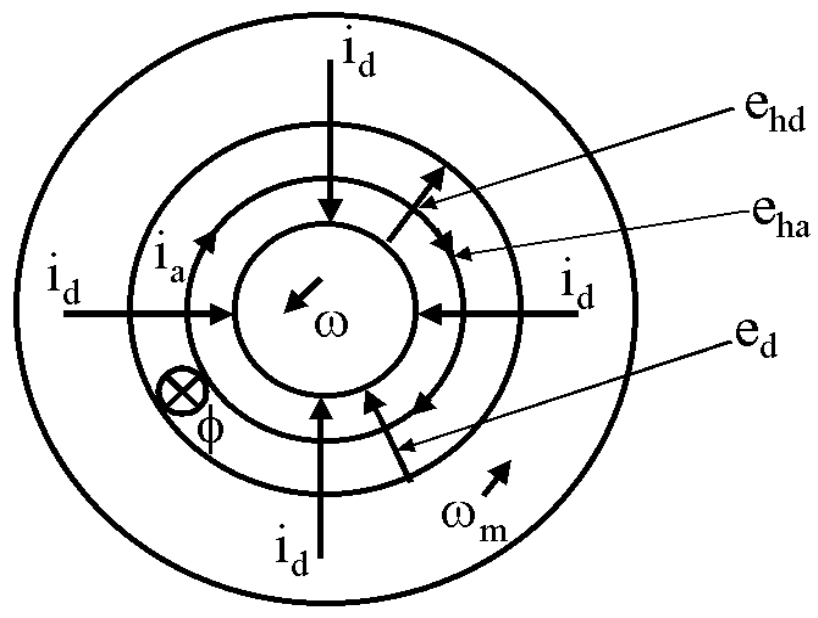

Fig. 2. Section through equatorial plane, from the north.

\section{The new theory and its basic equations}

The essence of the new theory is shown in Fig. 2, which depicts a section through the Earth in an equatorial plane perpendicular to the axis of spin of the metallic inner core. A very simplified picture of the current distributions is shown in Fig. 3, where the dynamo is viewed as a thick equivalent disk. The spin axis of the solid core is not aligned with that of the mantle, but the misalignment is ignored, for the moment. It is crucial for an explanation of time-varying declination, but does not affect the process of self-excitation. Initially, therefore, coaxial angular velocities $\omega_{m}$ and $\omega_{c}$ are assumed for mantle and solid core, respectively. With the reference directions shown for $\omega_{m}, \omega_{c}$, and for

$\omega=\omega_{c}-\omega_{m}$

in Fig. 2, $\omega_{m}$ has the value

$\omega_{m}=7.27 \times 10^{-5} \mathrm{rad} \mathrm{s}^{-1}$

The clockwise circulation of a current around the annulus causes a net downward magnetic flux $\Phi$. Since it is at a temperature well above the Curie point, and despite the extreme pressure, the core is likely to be paramagnetic rather than ferromagnetic (Van der Ziel, 1957). We invoke the linear relationship

$\Phi=L_{a} i_{a}$

where $L_{a}$ is the coefficient of self-inductance of the annulus.

The net inward dynamo emf, considered as generated in the fluid, is given by

$e_{d}=k \Phi \omega=k_{d} i_{a} \omega$

The net clockwise electromagnetic torque on the solid core is

$T_{e m}=k \Phi i_{d}=k_{d} i_{a} i_{d}$

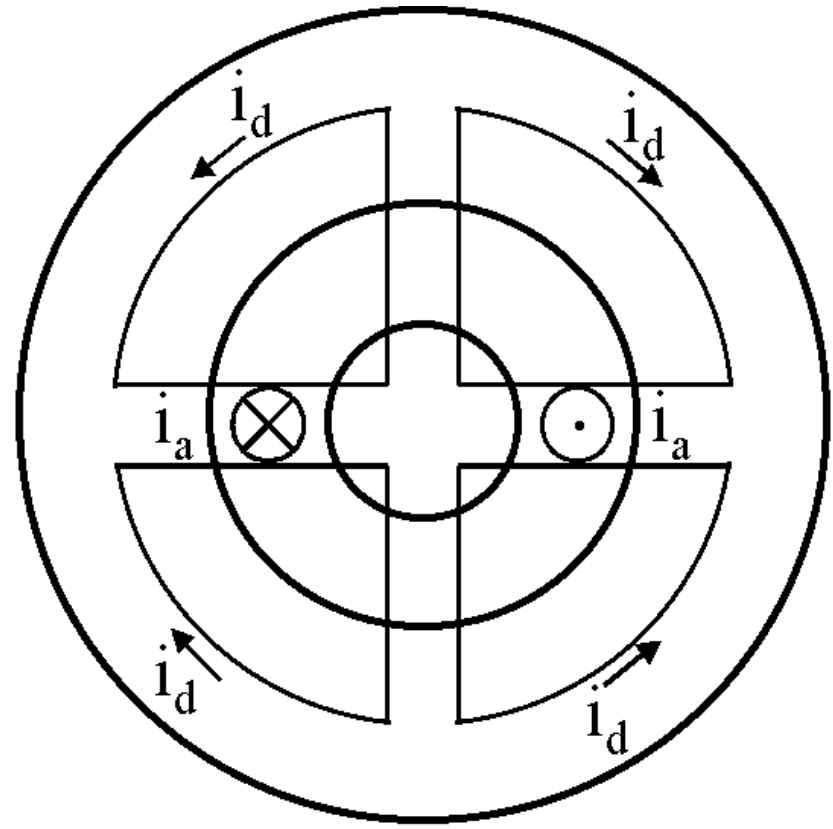

Fig. 3. Simplified side view of currents.

The Hall voltages indicated are

$e_{h a}=k_{h} i_{a} i_{d}$

$e_{h d}=k_{h} i_{a} i_{a}=k_{h} i_{a}^{2}$

If we denote the coefficient of self-inductance of the annulus by $L_{a}$; that of the dynamo armature circuit by $L_{d}$; the resistances of annulus and armature circuits by $R_{a}$ and $R_{d}$, respectively; the equivalent moments of inertia of the core and mantle by $J_{c}$ and $J_{m}$, respectively; an equivalent viscous friction coefficient for relative rotation of core and mantle by $F$; allow for Johnson-Nyquist thermal noise voltages $n_{d}(t)$ and $n_{a}(t)$, for a core-mantle interaction torque, $g(t)$, due to unspecified geophysical processes, and for an asteroid impact torque $h(t)$, the governing equations become:

$L_{a} \frac{d i_{a}}{d t}=-R_{a} i_{a}+k_{h} i_{a} i_{d}+n_{a}(t)$

$L_{d} \frac{d i_{d}}{d t}=-R_{d} i_{d}+k_{d} i_{a} \omega-k_{h} i_{a}^{2}+n_{d}(t)$

$J_{c} \frac{d \omega_{c}}{d t}=-F \omega-k_{d} i_{a} i_{d}+g(t)$

$J_{m} \frac{d \omega_{m}}{d t}=F \omega+k_{d} i_{a} i_{d}-g(t)+h(t)$

This constitutes a fourth-order nonlinear system, but the behaviour of the magnetic field is governed by a third-order subsystem with the fourth equation acting essentially as an observer (de Paor, 1998b): an equation through which some information regarding otherwise hidden variables may be inferred. In order to demonstrate this, we multiply Eq. (13) by $J_{m} /\left(J_{c}+J_{m}\right)$, Eq. (14) by $J_{c} /\left(J_{c}+J_{m}\right)$ and subtract the 
latter from the former, thus replacing Eq. (13) by

$J \frac{d \omega}{d t}=-F \omega-k_{d} i_{a} i_{d}+f(t)$

where

$J=\frac{J_{c} J_{m}}{J_{c}+J_{m}} \quad$ and $\quad f(t)=g(t)-\frac{J_{c}}{J_{m}+J_{c}} h(t)$

Eqs. (11), (12) and (15) govern the dynamics of the geomagnetic field, with Eq. (14) serving as an observer, in particular, for the torque $g(t)$. The mantle may be considered as the stator of an electrical machine, the solid core as the rotor and the fluid core as the winding, which, by virtue of the velocity gradient across it, can sustain a dynamo electromotive force and Hall voltages. The flux $\Phi$ is effectively locked to the stator. The above identifications of rotor, winding and stator will, for definiteness, be maintained in this exposition. It is appreciated, however, that the equations might apply to a situation in which the solid core remains the rotor, the winding is an as yet unidentified transition zone between solid and liquid cores, and the liquid core is the stator.

An extremely significant finding results from the autonomous system, Eqs. (11), (12) and (15) with $n_{a}(t)=$ $n_{d}(t)=f(t)=0$. Multiplying Eq. (12) by $i_{a}$, Eq. (13) by $i_{d}$ and Eq. (15) by $\omega$ and adding the results, all the electromagnetic and Hall terms cancel, to give:

$$
\begin{aligned}
0.5 \frac{d}{d t}\left(L_{a} i_{a}^{2}\right. & \left.+L_{d} i_{d}^{2}+J \omega^{2}\right) \\
= & -\left(R_{a} i_{a}^{2}+R_{d} i_{d}^{2}+F \omega^{2}\right)
\end{aligned}
$$

The term in the brackets on the left-hand side of Eq. (17) is a positive definite lyapunov function for the subsystem, with the negative definite time derivative on the right-hand side of Eq. (17) (Lyapunov, 1892; 1992). Thus, global asymptotic stability of the null state is proved; chaos is not intrinsic: it can only be introduced by forcing terms, which might, indeed, induce parameter variation. The autonomous subsystem would be inexorably run down to the null state.

It is convenient to write the equations for the third-order subsystem in dimensionless form. This yields significant insight into the parameter groups responsible for the essential nature of the behaviour. Eqs. (11), (12) and (15) become:

$$
\begin{aligned}
& \frac{d x_{1}}{d \tau}=\eta\left(-x_{1}+x_{1} x_{2}+\mu n_{a}^{+}(\tau)\right) \\
& \frac{d x_{2}}{d \tau}=\frac{1}{\eta}\left(-x_{2}+2 x_{3} x_{1}-x_{1}^{2}+\zeta n_{d}^{+}(\tau)\right) \\
& \frac{d x_{3}}{d \tau}=\eta \gamma\left(-x_{3}-\delta x_{1} x_{2}+\epsilon f^{+}(\tau)\right)
\end{aligned}
$$

where

$$
\begin{aligned}
& x_{1}=\frac{k_{h}}{\sqrt{R_{a} R_{d}}} i_{a}, \quad x_{2}=\frac{k_{h}}{R_{a}} i_{d}, \quad x_{3}=\frac{k_{d}}{2 \sqrt{R_{a} R_{d}}} \omega \\
& \tau=\sqrt{\frac{R_{a} R_{d}}{L_{a} L_{d}}} t, \quad \eta=\sqrt{\frac{R_{a} L_{d}}{L_{a} R_{d}}}, \quad \gamma=\frac{L_{a} f}{R_{a} J} \\
& \delta=\frac{k_{d}^{2} R_{a}}{2 k_{h}^{2} F}, \quad \epsilon=\frac{k_{d}}{2 F \sqrt{R_{a} R_{d}}}, \quad \mu=\frac{k_{h}}{R_{a} \sqrt{R_{a} R_{d}}} \\
& \zeta=\frac{k_{h}}{R_{a} R_{d}}
\end{aligned}
$$

We now explore the dynamics of Eqs. (18), (19) and (20) with the forcing functions initially set to zero, and subsequently, readmitted for specific purposes.

\section{Self-excitation of the field}

It was pointed out above that in the absence of forcing functions, the third-order subsystem governing the geomagnetic field would collapse into the null state. That does not imply that it is without interesting dynamics. Estimation of parameters indicates that $\omega$ varies on a much slower time scale than $i_{a}$ and $i_{d}$, so that we can justifiably consider the solutions of Eqs. (18) and (19) over a time frame in which $x_{3}$ is substantially constant. In such a circumstance, the autonomous versions of Eqs. (18) and (19) have three pseudo equilibrium states:

$$
\begin{array}{lll}
P_{0}: & x_{1}=0 ; & x_{2}=0 \\
P_{1}: & x_{1}=x_{3}\left(1-\sqrt{1-x_{3}^{-2}}\right) ; & x_{2}=1 \\
P_{2}: & x_{1}=x_{3}\left(1+\sqrt{1-x_{3}^{-2}}\right) ; & x_{2}=1
\end{array}
$$

In the following remarks we consider $x_{3}>1$. The dynamics for $x_{3}<-1$ are the image in the $x_{2}$ axis.

$P_{0}$ is the unexcited state. Perturbation analysis reveals that it possesses local asymptotic stability irrespective of the value of $x_{3}$. It is a stable node, characterised by two negative, real eigenvalues.

$P_{1}$ and $P_{2}$ exist as real states only for $x_{3}^{2} \geq 1$.

For $x_{3}^{2}>1 P_{1}$ is a saddle point, characterised by one real, positive eigenvalue and one real, negative eigenvalue. There are two singular trajectories along which motion is attracted to $P_{1}$. These, taken together, constitute a separatrix, whose role is profound.

When $P_{2}$ first appears, it is a stable node, but it changes to a stable focus for $x_{3}$ just a little greater than one.

The separatrix is the boundary between the domains of attraction of $P_{0}$ and $P_{2}: P_{0}$ consists of the whole of the left half of the $\left(x_{1}, x_{2}\right)$ plane, and the portion of the right half plane lying to the left of the separatrix, while $P_{2}$ consists of the portion of the right half plane lying to the right of the separatrix.

The above observations are illustrated in Fig. 4, which has been drawn, only for illustration purposes, for $x_{3}=10$. (The 


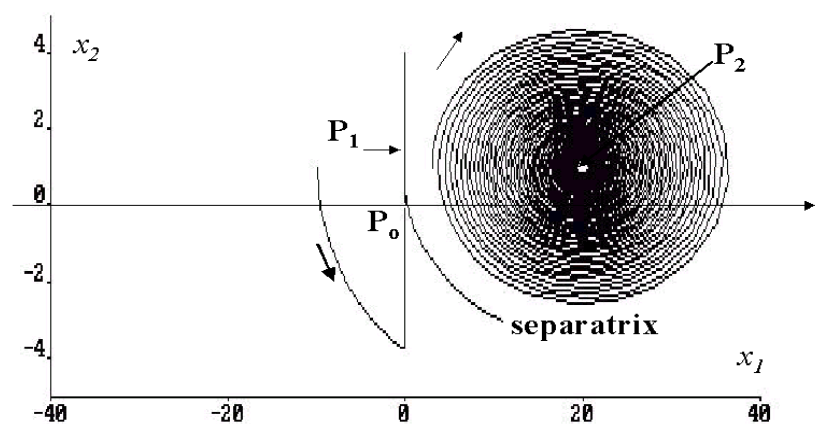

Fig. 4. Trajectories in the $\left(x_{1}, x_{2}\right)$ plane.

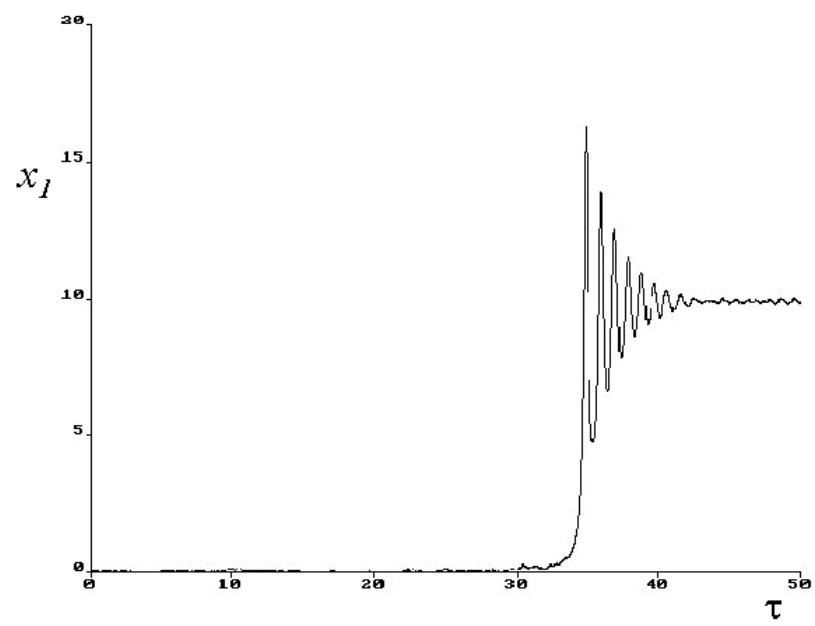

Fig. 5. Self-excitation triggered by thermal noise voltage.

value $x_{3}=600$ which is actually used in the analysis of secular variations gives such a low damping ratio on the spiral that its turns cannot be distinguished on a graph). In order to produce excitation of the dipolar geomagnetic field, the system must make the transition from $P_{0}$ across the separatrix to the right. This has to be achieved by admitting the forcing functions $n_{a}(\tau)$ and $n_{d}(\tau)$. An excitation experiment is sketched in Fig. 5, under the influence of a thermal noise voltage $n_{a}(\tau)$ acting alone, again drawn for $x_{3}=10$.

As $x_{3}$ increases, the separatrix is forced back onto the $x_{2}$ axis, closer to $P_{0}$, and self-excitation becomes possible with a smaller noise signal.

\section{Reversal of the field}

If $\omega$, and thus, $x_{3}$ changes sign, the whole trajectory pattern shown in Fig. 4 is reflected in the $x_{2}$ axis. Herein lies one possibility of field reversal. In order to illustrate this, we perform a thought experiment on Eq. (20).

We imagine that, on a geological time scale, $g(t)$ constitutes a very rapid pulse. This would represent a major coremantle interaction torque. The case of $h(t)$ being an impulse

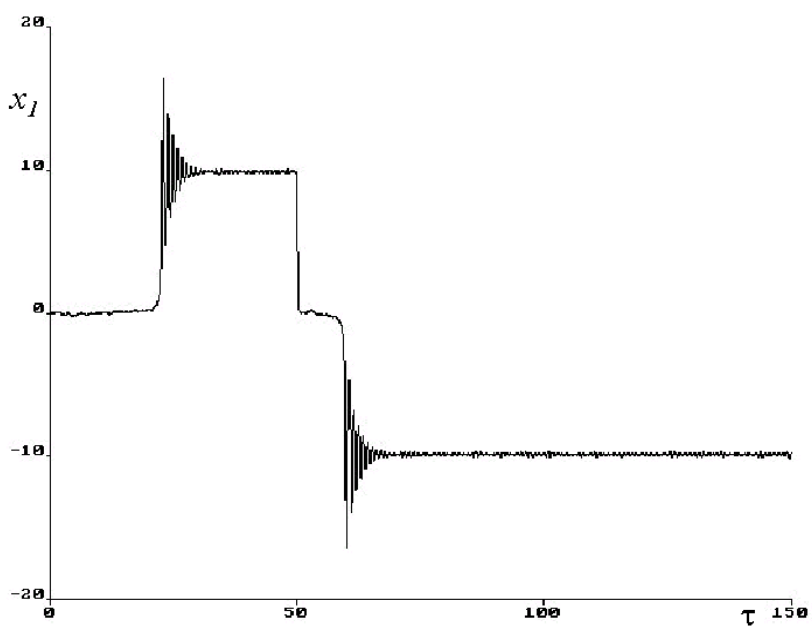

Fig. 6. Field reversal following reversal of $\omega$.

might be an asteroid impact of such magnitude as to leave its mark on geophysical processes, such as climate, with possible major consequences for patterns of life.

Subject to such an assumed $g(t)$, we model $f^{+}(\tau)$ as a Dirac delta function of strength $k$ occurring at the dimensionless time $\tau_{0}$ :

$f^{+}(\tau)=k \delta\left(\tau-\tau_{0}\right)$

Integrating Eq. (20) over an infinitesimal range across $\tau=\tau_{0}$ and rearranging gives

$x_{3}\left(\tau_{0}^{+}\right)=x_{3}\left(\tau_{0}^{-}\right)+\frac{2 \epsilon \eta \gamma k}{\beta}$

Thus, to reverse the field exactly by choosing $x_{3}\left(\tau_{0}^{+}\right)=$ $-x_{3}\left(\tau_{0}^{-}\right)$, we require

$k=-\frac{\beta x_{3}\left(\tau_{0}^{-}\right)}{\epsilon \eta \gamma}$

The result of applying this impulse to Eq. (20) and plotting the resulting behaviour of $x_{1}(\tau)$ is shown in Fig. 6. After $x_{3}$ has changed sign, the system awaits a perturbation of sufficient magnitude, induced in this experiment by the noise signal $n_{a}^{+}(\tau)$, in order to self-excite again.

It is interesting to contemplate the change in length of day associated with internally induced field reversal. The third member of Eq. (21) gives

$\omega=2 \frac{\sqrt{R_{a} R_{d}}}{k_{d}} x_{3}$

Assuming that the relative permeability of the core is $\mu_{r} \approx$ 1 , it is estimated using results from the electromagnetic analysis section below that, given a dimensionless parameter $\lambda$ equal to unity and a quadratic fall-off in fluid angular velocity with radius, $k_{d} \approx 6.21 \times 10^{-1} \mathrm{H}$. Taking the electrical conductivity of the fluid core as $\sigma \approx 10^{-7} \mathrm{Sm}^{-1}$ (typical of molten metals (Inglis, 1955), but used with great caution), 


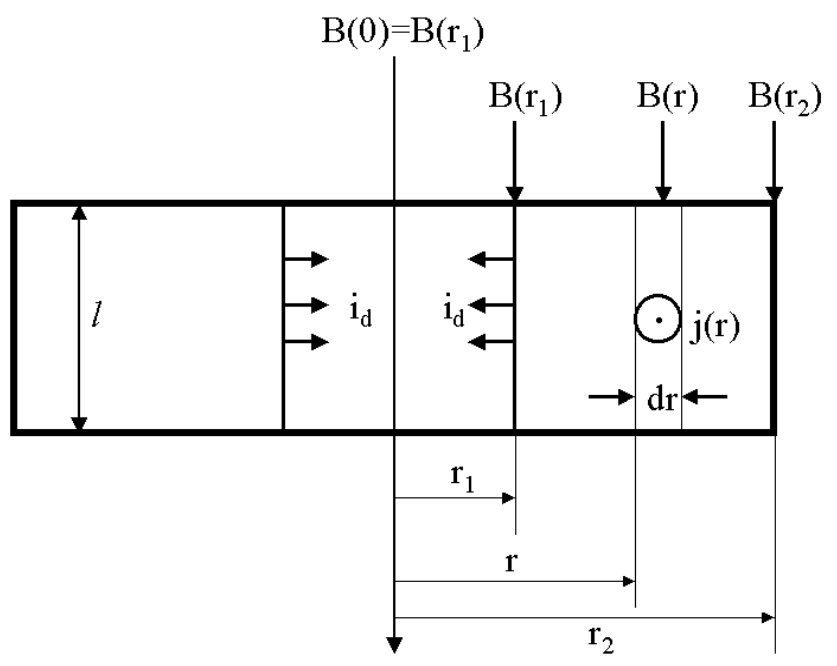

Fig. 7. Geometry for calculation of $k_{h}, k_{d}$ and $R_{a}$.

we estimate $R_{a} \approx 1.72 \times 10^{-13} \Omega$. Taking $R_{d} \approx 0.1 R_{a}$, we find that for $x_{3}=600$,

$\omega\left(\tau_{0}^{-}\right) \approx 8.3 \times 10^{-11} \mathrm{rad} \mathrm{s}^{-1}$

currently estimated by Vidale et al. (2000).

From Eqs. (14) and (15) we have the relation that changes in $\omega$ and $\omega_{m}$ due to an impulse $g(t)$ are related by

$\Delta \omega_{m}=-\frac{J_{c}}{J_{c}+J_{m}} \Delta \omega$

Taking $J_{c} \approx 0.01 J_{m}$ (so high on account of entrained fluid), a change of $1.66 \times 10^{-10} \mathrm{rad} \mathrm{s}^{-1}$ in $|\omega|$ is accompanied by a change of $8.3 \times 10^{-13} \mathrm{rad} \mathrm{s}^{-1}$ in $\left|\omega_{m}\right|$. In view of Eq. (5), this represents a change of about 0.001 seconds in length of day.

The above discussion refers only to one possible mechanism of field reversal. It seems to the author that there could well be another: reversal of the sign of the Hall coefficient. This would be achieved by conduction type transition (from $p$-type to $n$-type and vice versa), a phenomenon akin to population inversion in laser action. Given the extreme conditions of temperature and pressure in the core, this might be induced by shock waves, but much work remains to be done to explore the idea.

\section{Electromagnetic theory of the model under pseudo equilibrium conditions}

This section presents electromagnetic analysis of pseudo equilibrium conditions in the model. This leads to calculation of $k_{h}, k_{d}, R_{a}, R_{d}$ and $L_{a} . L_{d}$ has not yet been evaluated. The method of attack introduces a parameter $\lambda$ on which various quantities depend, and this is evaluated as 1 . In this section, the symbols $i_{a}$ and $i_{d}$ refer not to general time variations as in Eqs. (11) to (14), but to pseudo equilibrium values.

Purely for tractability, the analysis is based on a cylindrical approximation to the actual spherical geometry, suggested by the simplified current distribution sketched in Fig. 3. This carries the hint that it might be valid to regard the solid core as functioning primarily as a collecting and distributing electrode for the dynamo current $i_{d}$. The solid core is here modelled as a cylinder, whose height $l$ is chosen so that its curved area is equal to the surface area of the sphere. This criterion of equivalence gives the length of the cylinder as

$l=2 r_{1}$

where $r_{1}=1.25 \times 10^{6} \mathrm{~m}$ (Inglis, 1955).

We now have the geometry shown in Fig. 7, which models the central band from Fig. 3. The outer radius of the fluid annulus is $r_{2}=3.4 \times 10^{6} \mathrm{~m}$ (Inglis, 1955).

The fluid annulus is the seat of dynamo action and of currents driven by the Hall effect in the right-hand screw direction about the geomagnetic axis.

The Hall voltage per unit distance, acting to oppose the inward dynamo current $i_{d}$, with the reference directions indicated, is given by

$E_{d}(r)=R_{h} B(r) j(r)$

and the Hall voltage per unit distance, acting to drive current of density $j(r)$ around the annulus in the right-hand screw direction is

$E_{a}(r)=R_{h} B(r) \frac{i_{d}}{2 \pi r l}$

The relation

$j(r)=\sigma E_{a}(r)$

converts Eq. (31) to

$r j(r)=\frac{R_{h} \sigma i_{d}}{2 \pi l} B(r)$

where $\sigma$ is the electrical conductivity of the fluid.

Ampere's Circuital Theorem (Ohanian, 1988) gives

$\frac{B(r)}{\mu_{0}}=\frac{B\left(r_{2}\right)}{\mu_{0}}+\int_{r}^{r_{2}} j(y) d y$

Inserting Eq. (34) into Eq. (33) leads to an integral equation governing $j(r)$ :

$r j(r)=\lambda\left(\frac{B\left(r_{2}\right)}{\mu_{0}}+\int_{r}^{r_{2}} j(y) d y\right)$

where the positive, dimensionless parameter $\lambda$ is defined by

$\lambda=\frac{R_{h} \mu_{0} \sigma i_{d}}{2 \pi l}$

with $\mu_{0}=4 \pi \times 10^{-7} \mathrm{H} \mathrm{m}^{-1}$ being the magnetic permeability of free space. The solution of Eq. (35), subject to a study of its boundary conditions, has been obtained as

$j(r)=\frac{i_{a} \lambda}{l r_{1}}\left(1-\beta^{-\lambda}\right)^{-1}\left(\frac{r_{1}}{r}\right)^{\lambda+1}$

where

$\beta=\frac{r_{2}}{r_{1}}$ 
Because of the spatial current density distribution enshrined in Eq. (37), calculation of the equivalent lumped resistance $R_{a}$ of the annulus has to proceed by evaluating the Ohmic power dissipated in the annulus and equating it to $i_{a}^{2} R_{a}$. This Ohmic power is given by

$p_{a}=\frac{2 \pi l}{\sigma} \int_{r_{1}}^{r_{2}} r j^{2}(r) d r$

Substituting Eq. (37) into Eq. (39), integrating and dividing by $i_{a}^{2}$ leads to the result:

$R_{a}=\frac{\pi \lambda}{\sigma l} \frac{1-\beta^{-2 \lambda}}{\left(1-\beta^{-\lambda}\right)^{2}}$

Integrating Eq. (30) from $r=r_{1}$ to $r=r_{2}$, subject to Eqs. (33) and (37), yields an expression for the Hall back emf $e_{h d}$ in the dynamo circuit. Dividing this by $i_{a}^{2}$, as indicated by Eq. (12), leads to

$k_{h}=\frac{\pi \lambda^{2}}{i_{d} \sigma l} \frac{1-\beta^{-2 \lambda}}{\left(1-\beta^{-\lambda}\right)^{2}}$

Eq. (11) indicates that under pseudo-equilibrium in the excited state $\left(i_{a} \neq 0\right)$,

$i_{d}=\frac{R_{a}}{k_{h}}$

We now rewrite Eq. (41) as

$k_{h}=\frac{\mu_{0} R_{h}}{2 l^{2}} \lambda \frac{1-\beta^{-2 \lambda}}{\left(1-\beta^{-\lambda}\right)^{2}}$

revealing the dependence of $k_{h}$ on the Hall coefficient.

We now calculate the dynamo constant $k_{d}$. Assume that at radius $r$ is out from the centre, and the fluid is circulating at angular velocity $\Omega(r)$, net dynamo electromotive force is

$e_{d}=\int_{r_{1}}^{r_{2}} B(r) r \Omega(r) d r$

Clearly, $\Omega\left(r_{1}\right)=\omega$ (the angular velocity of the central electrode relative to the mantle) and $\Omega\left(r_{2}\right)=0$, with $\Omega(r)$ decreasing monotonically between those limits. A simple functional form to describe this is

$\Omega(r)=\omega\left(\frac{r_{2}-r}{r_{2}-r_{1}}\right)^{v}, \quad v \geq 1, r_{1} \leq r \leq r_{2}$

but that is offered just for illustration purposes.

Using $B(r)$ from Eq. (33), with $j(r)$ then furnished by Eq. (37), Eq. (44), divided through by $i_{a} \omega$, in accordance with Eq. (7), yields

$k_{d}=\frac{\mu_{0} r_{1}^{2}}{l\left(1-\beta^{-\lambda}\right)} \int_{1}^{\beta} g(x) x^{-\lambda+1} d x$

with, in agreement with Eq. (45),

$g(x)=\left(\frac{\beta-x}{\beta-1}\right)^{v}, \quad 1 \leq x \leq \beta$

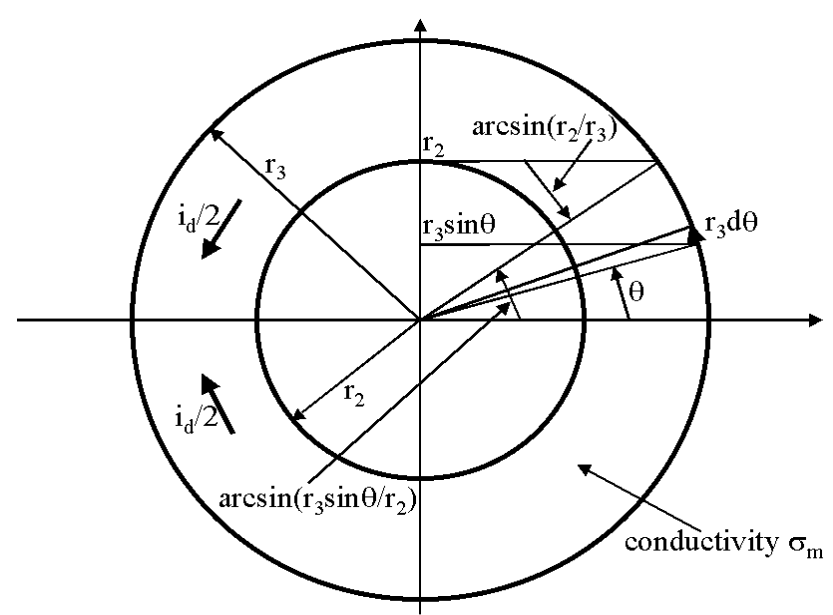

Fig. 8. Geometry used for estimation of $R_{d m}$.

The resistance $R_{d}$ in the dynamo circuit has three additive components: $R_{d a}$ is due to inward current flow across the fluid annulus in Fig. 7; $R_{d r}$ to the return paths downward and upward to the central collecting electrode in Fig. 7; and $R_{d m}$ to the current flow through the mantle, as indicated in Fig. 3. The first two components are very easy to calculate:

$R_{d a}=\frac{\ln (\beta)}{2 \pi \sigma l}$

and

$R_{d r}=\frac{\sqrt{r_{3}^{2}-r_{1}^{2}}-l / 2}{2 \pi \sigma r_{1}^{2}}$

where it has been assumed that the collecting cylinder extends to touch the surface of the Earth. This overestimation is to compensate for the underestimation of $R_{d m}$.

Partial evaluation of $R_{d m}$ is based on the geometry defined in Fig. 8, from which, considering only the contribution up to height $r_{2}$, we obtain;

$R_{d m}=\frac{r_{3} \arcsin \left(r_{3} / r_{2}\right)}{2 \pi \sigma_{m}\left(r_{3}^{2}-r_{2}^{2}\right)}$

where $\sigma_{m}$ is the effective conductivity of the mantle.

We now evaluate $\lambda$ by considering the dimensionless form of the equations of motion, given by Eqs. (18), (19) and (20), along with the scaling factors given in Eq. (21). The second of these gives $i_{d}=\left(R_{a} / k_{h}\right) x_{2}$. Since the pseudo equilibrium value of $x_{2}$ is 1 , Eqs. (40) and (41) yield

$i_{d}=\frac{\pi \lambda}{\sigma l} \frac{i_{d} \sigma l}{\pi \lambda^{2}}$

There follows at once

$\lambda=1$ 


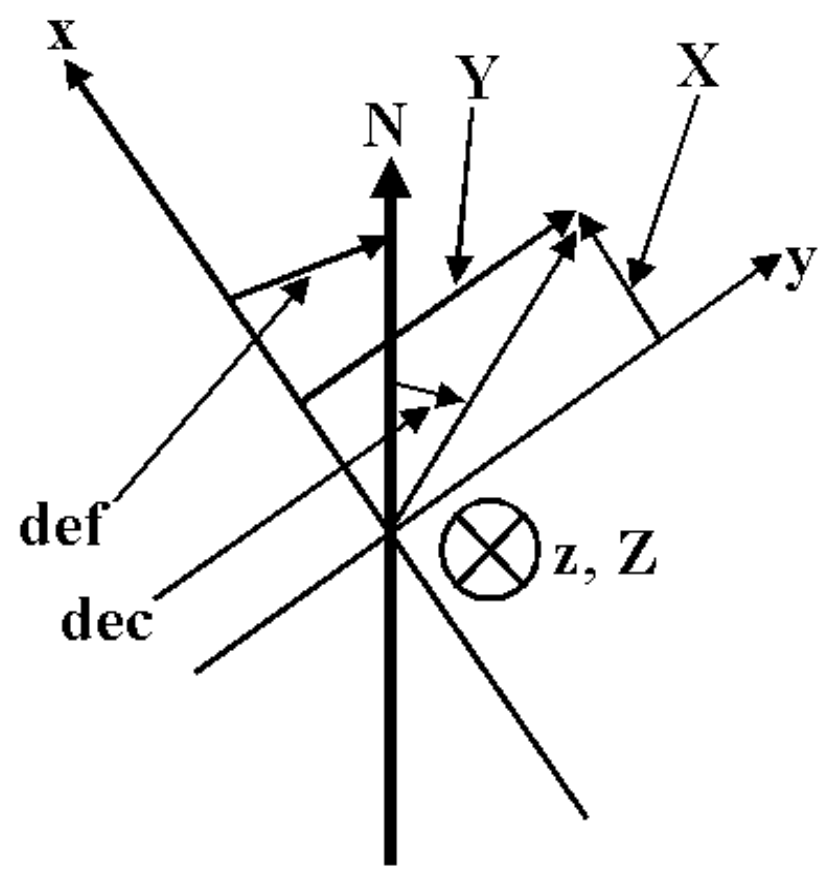

Fig. 9. Geometry used to calculate dip and declination.

The expressions for the parameters evaluated so far now simplify to:

$$
\begin{aligned}
R_{a} & =\frac{\pi}{\sigma l} \frac{r_{1}+r_{2}}{r_{2}-r_{1}} \\
k_{h} & =\frac{\mu R_{h}}{2 l^{2}} \frac{r_{1}+r_{2}}{r_{2}-r_{1}} \\
k_{d} & =\frac{\mu_{0} r_{1}^{2} r_{2}}{l\left(r_{2}-r_{1}\right)} \int_{1}^{\beta} g(x) d x \\
R_{d} & =\frac{1}{2 \pi \sigma}\left(\frac{\ln \left(r_{2} / r_{1}\right)}{l}+\frac{\sqrt{r_{3}^{2}-r_{1}^{2}-l / 2}}{r_{1}^{2}}\right. \\
& \left.+\frac{r_{3} \arcsin \left(r_{3} / r_{2}\right)}{r_{3}^{2}-r_{2}^{2}} \frac{\sigma}{\sigma_{m}}\right)
\end{aligned}
$$

Substituting Eq. (37) into Eq. (33) in order to evaluate $B(r)$, integrating that over the annulus and dividing by $i_{a}$ gives

$L_{a}=\frac{2 \mu_{0} r_{1} r_{2}}{l}$

The first member of Eq. (21), taken in conjunction with Eqs. (22) and (38), yields the pseudo equilibrium value of the polar geomagnetic field:

$B_{p}=2 \pi b \chi x_{3} \frac{1+\sqrt{1-x_{3}^{-2}}}{\sigma R_{h}\left(1-\beta^{-1}\right)}$

where

$b=\sqrt{\frac{R_{d}}{R_{a}}}$ and

$\chi=\frac{B_{p}}{B\left(r_{1}\right)}$

$\chi$ has been evaluated for the author as 0.297 .

It may seem surprising that $B_{p}$ should be inversely proportional to $\sigma R_{h}$, and yet, dimensional analysis makes that inevitable. $B_{p}$ has the dimensions of $\mathrm{V} \mathrm{s} \mathrm{m}^{-2}$. Eq. (2) shows that $\sigma R_{h}$ has the dimensions of carrier mobility, i.e. $\left[\mathrm{m} \mathrm{s}^{-1}\right] /$ $\left[\mathrm{V} \mathrm{m}^{-1}\right]=\mathrm{V}^{-1} \mathrm{~s}^{-1} \mathrm{~m}^{2}$, the inverse dimensions of magnetic flux density. $B_{p}$ is seen to increase with the normalised angular velocity of the solid core relative to the mantle, i.e. with $\omega=2 x_{3} \sqrt{R_{a} R_{d}} / k_{d}$, once the condition $x_{3}^{2}>1$ is satisfied. The first two members of Eq. (21) show that the pseudo equilibrium values of $i_{a}$ and $i_{d}$ vary inversely with the Hall coefficient: the larger $R_{h}$, the smaller the current $i_{d}$ needed to sustain the field.

With regard to quantities occurring in Eq. (57), it is almost certain that the current value of polar flux density, $B_{p} \approx$ $6.24 \times 10^{-5} \mathrm{~T}$ (Lee, 1963) is not the pseudo equilibrium value. Rather, it might be possible to estimate that from the pattern of variation in the magnitude of the Earth's magnetic field, observed at some definite location over the past few centuries, extrapolated to the location of one of the poles. As far as $\sigma R_{h}$ is concerned, there are competing influences at play (Inglis, 1955), such as disorder due to high temperature and order due to high pressure. It may even be that, as observed in the Hall effect at extremely low temperatures and normal pressures, unexpected phenomena, such as electron spin coupling and carrier aggregation, could dominate the behaviour.

\section{$7 \quad$ Analysis of secular variations}

There is a strong dynamic pointer to the plausibility of the present theory lying in the state plane plot in Fig. 4. The quantities $x_{1}$ and $x_{2}$ are scaled versions of the annulus current and the dynamo current, respectively. The dipolar component of geomagnetic field flux density is generated by $i_{a}$. If $i_{d}$ were symmetrically distributed with respect to the geographic axis of a perfectly homogeneous Earth, Ampere's Circuital Theorem applied around a circle of latitude shows that it would have no magnetic consequence at the surface. However, displacement of the magnetic axis from the geographic North-South axis is, as the author has reasoned and confirmed by experiments on a physical model of the current distribution presented here, sufficient to introduce a transverse magnetic field due to $i_{d}$. (Another transverse magnetising possibility would be provided if a fraction of $i_{d}$ were to flow between magnetic equator and poles through the atmosphere. This would accord with a suggestion which the author recalls being ascribed to Ampere, and might well shed some light on diurnal and seasonal components of variation). We propose that the interaction between $i_{d}$ and $i_{a}$ is being observed in secular variations, with a single cycle around the spiral taking 624 years (see Fig. 4). 
In order to make the above observations concrete, tentative models of secular variation at London, the Cape of Good Hope and Palermo are presented, based on data gathered over the past four hundred years. Our task is to identify the value of $x_{3}$ which sets the damping ratio of the spiral; an appropriate starting point $\left(x_{10}, x_{20}\right)$, which corresponds to conditions anywhere on Earth in a datum year which, for a special reason, is taken as 1795; the parameter $\eta$ in Eqs. (18), and (19); and physically reasonable, location-specific functions of $x_{1}$ and $x_{2}$ to govern the three orthogonal components of the field.

The geometry for calculating dip and declination (abbreviated to dec in the equations) is shown in Fig. 9. The $(x, y)$ plane is horizontal. The $x$ axis points in the direction indicated by a compass needle, in the case that the field lies in the plane containing the Earth's magnetic poles and the location in question. This is local dipolar magnetic north. We have derived an equation for the deflection (abbreviated def), which is the angle between geographic north and the $x$ axis. The angle is positive if geographic north lies east of $x$. (It is used to calculate def here, but it is so involved that its publication is deferred.) The $z$ axis points directly downwards. In this initial treatment, the $x$ component of the field is assumed to be directly proportional to $x_{1}$, although one realizes that this must be an approximation. The $y$ component is taken to be a function of $x_{2}$, and all magnetic nonlinearity in the calculation of declination is lumped into it. The $z$ component is taken to depend linearly on $x_{1}$, and nonlinearly on $x_{2}$. Hysteresis has been identified in the relations between the $y$ and $z$ components, and $x_{2}$. Closed minor hysteresis loops, relating the $y$ component to $x_{2}$, are generated by ad hoc expressions $f\left(x_{2}\right)$, and the $z$ component is treated more simply, but seemingly quite accurately, by incorporating terms linear in $d x_{2} / d \tau$.

The notation used in this section is only applicable here. Expressions for the three orthogonal components of the geomagnetic field are:

$$
\begin{aligned}
& X=g x_{1} \\
& Y=g f\left(x_{2}\right) \\
& Z=g\left(a x_{1}+b x_{2}+c \frac{d x_{2}}{d \tau}\right)
\end{aligned}
$$

The expressions for dec and dip are

$$
\begin{aligned}
& \operatorname{dec}=\tan ^{-1}(Y / X)-\operatorname{def} \\
& \operatorname{dip}=\tan ^{-1}\left(\frac{Z}{\sqrt{X^{2}+Y^{2}}}\right)
\end{aligned}
$$

Starting with London in 1795 (the differential equations are integrated forward and backward from this datum), we have, after much experimentation, zeroed in on the following quantities. They are offered tentatively:

$x_{3}=600$

$\left(x_{10}, x_{20}\right)=(1200,0)$

$\eta=1$

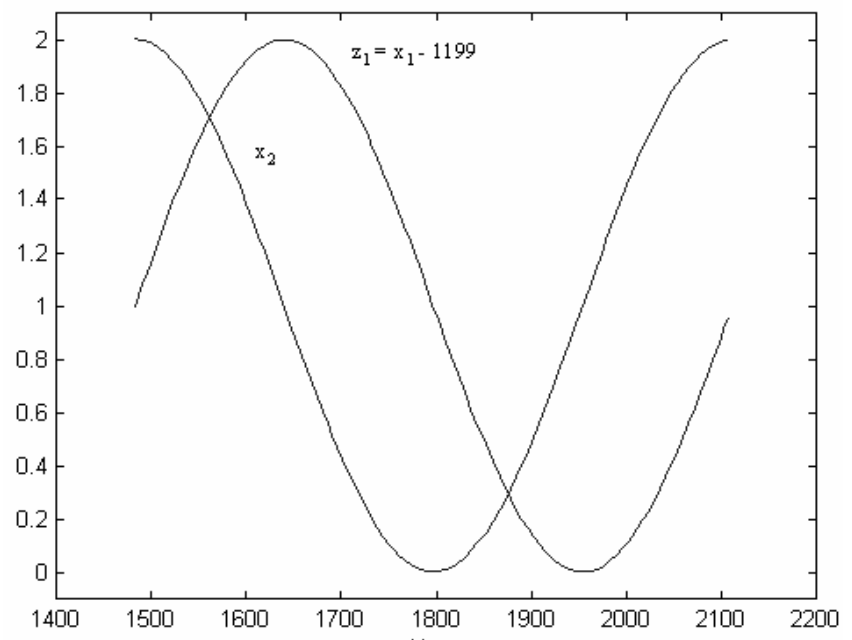

Fig. 10. Graphs of $\left[x_{1}-1199\right]$ and $x_{2}$ versus year.

Time-scaling makes $\tau=1$ correspond to 120000 years.

The resulting graphs of $x_{1}-1199$ and $x_{2}$, as functions of year, are shown in Fig. 10. These are taken to apply at all points on Earth.

The values of def have been computed using the formula alluded to above, followed by experimentation to compute the parameter values in $f\left(x_{2}\right)$ to fit declination, and a Gaussian least squares error technique, to find the $a, b$ and $c$ values which best fit dip.

The relations used for London are

$f\left(x_{2}\right)=\left\{\begin{array}{lll}f_{d}\left(x_{2}\right) & \text { for } \frac{d x_{2}}{d \tau}<0 \text { (between } 1483 \text { and 1795) } \\ f_{u}\left(x_{2}\right) & \text { for } \frac{d x_{2}}{d \tau}>0 \text { (between } 1795 \text { and 2107), }\end{array}\right.$

where

$f_{u}\left(x_{2}\right)= \begin{cases}309 x_{2} & \text { for } x_{2}<1.45 \\ 309 x_{2}+1200\left(x_{2}-1.45\right)^{2} & \text { for } x_{2}>1.45\end{cases}$

and

$f_{d}\left(x_{2}\right)=325 x_{2}+340\left(1-\exp \left(-x_{2} / 0.4\right)\right)$,

along with

$\operatorname{def}=24.05^{\circ}, \quad a=2.99, \quad b=116.91, \quad c=-0.7286$.

The fact that def $=24.05^{\circ}$ at London suggested taking 1795 as time origin, for the declination in London, in that year, was almost exactly equal to minus that value, while the rate of change of declination was zero. This suggested that both $x_{2}$ and $\mathrm{d} x_{2} / \mathrm{d} \tau$ were zero in 1795 . Corroboration was obtained at Palermo, and at several other sites studied since the preparation of this paper, although it was necessary to introduce an offset in $Y$ at the Cape of Good Hope.

Some results are given below, with observations taken from Malin and Bullard (1981), except for the declination at 2000.5 , which was supplied by an airline pilot. All angles are in degrees. 


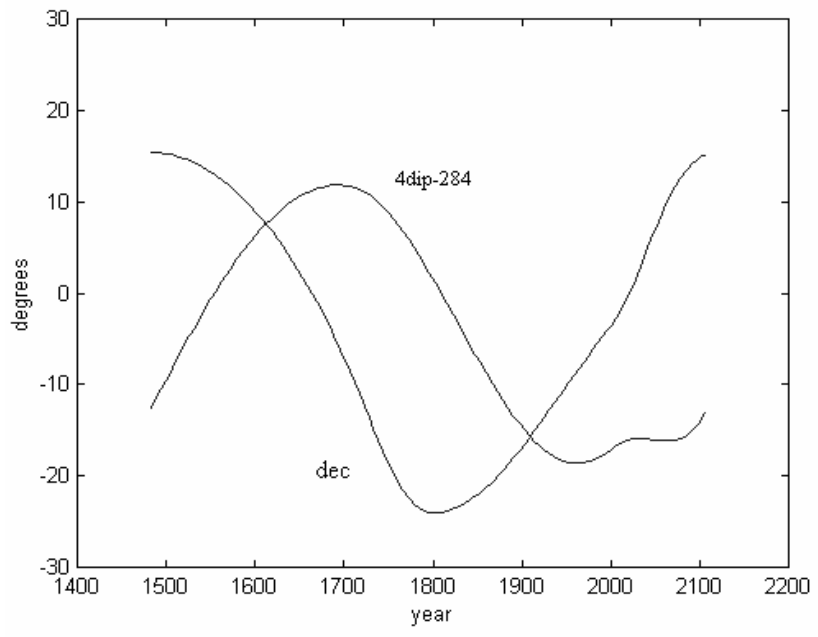

Fig. 11. Declination and [4dip-284] at London versus year.

First, we have triples giving year, observed declination and computed declination: 1576, 11.5, 11.13; 1657.5, 0 , $1.06 ; 1698.8,-7,-7.03 ; 1745.2,-17,-17.93 ; 1799.6$, $24.02,-23.99 ; 1809.5,-24.183,-23.89 ; 1823.5,-24.383$, $-23.46 ; 1857,-21.767,-21.3 ; 1900.5,-16.483,-16.62$; $1925.5,-13.378,-13.3 ; 1948.7,-9.617,-10.08 ; 1960.5$, $-8.518,-8.46 ; 1975.5,-7.155,-6.45 ; 2000.5,-3.5$, -3.38 .

Next, we have triples giving year, observed dip and computed dip: $1576.5,71.833,71.883 ; 1673,73.783,73.896$; $1719,73.75,73.802 ; 1750.5,73.5,73.142 ; 1790,71.895$, $71.715 ; 1828.6,69.783,70.057 ; 1850.5,68.782,69.107$; $1890.6,67.505,67.568 ; 1910.5,66.88,69.989 ; 1956.5$, $66.623,66.348 ; 1975.5,66.532,66.404$.

Graphs of declination and [4dip-284] versus year at London, produced by simulation, are plotted in Fig. 11. The anticlockwise loop of dip vs. declination, plotted in Fig. 12, entered in 1483, comes closest to closing in 2107.

With regard to declination at London, a quotation from Malin and Bullard (1981) may be of interest:

"It happens that the changes in declination at London have been exceptionally large and are of considerable interest for the dynamo theory of the origin of the field and its changes. How could the declination have been $11^{\circ} \mathrm{E}$ in the late 16th century, at a time when the north magnetic dip-pole and the dipole axis were not far from their present positions to the north of Hudson's Bay? The subsequent swing of the field to $24^{\circ}$ west in 1820 , still without any substantial shift of the pole, is equally remarkable. Presumably an exceptionally large eddy or magneto-dynamic wave passed under Europe from east to west, near the surface of the Earth's core."

By the same logic, an eddy from west to east would have been necessary for the eastward trend of declination, but we hope to have demonstrated that eddies are not the cause of time-varying declination.

At the Cape of Good Hope, Brewster (1838) gives twelve

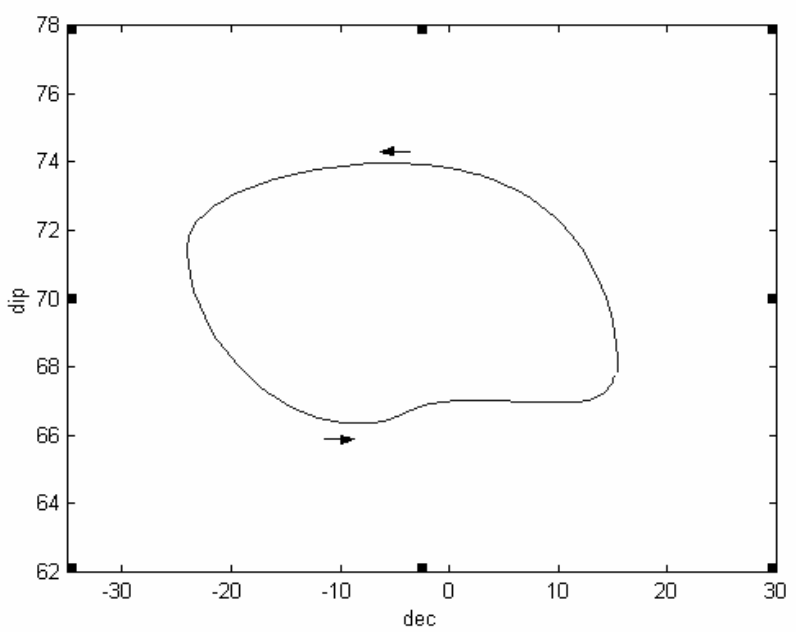

Fig. 12. Dip vs. declination at London.

observations of declination. We have augmented them with a value for 1846.3, obtained from the British Geological Survey, and fitted the data with the model

$f_{d}=-265.8+210 x_{2}+300\left(1-\exp \left(-x_{2} / 0.3\right)\right)$

$f_{u}=-265.8+195\left(x_{2}-0.08\right)^{2}$

$\operatorname{def}=14.617^{\circ}$

which gives the following triples: $1605,0.5,-.02 ; 1609$, $-0.2,-0.58 ; 1614,-1.5,-1.06 ; 1667,-7.25,-7.19$; $1675,-8.5,-8.32 ; 1702,-12.833,-12.69 ; 1724,-16.45$, $-16.96 ; 1752,-19,-22.6 ; 1768,-19.5,-25.19 ; 1775$, $-21.233,-26.03 ; 1791,-25.667,-27.07 ; 1804,-25.067$, $-27.11 ; 1846.3,-29.145,-27.09$.

Some historical data for Palermo are on the Internet site www.ingrm.it/geomag/palermo.htm. These are modelled by

$f_{u}\left(x_{2}\right)= \begin{cases}360 x_{2} & \text { for } \quad x_{2}<1.61 \\ 360 x_{2}+1200\left(x_{2}-1.61\right)^{2} & \text { for } \quad x_{2}>1.61\end{cases}$

and

$f_{d}\left(x_{2}\right)=360 x_{2}+190\left(1-\exp \left(-x_{2} / 0.4\right)\right)$,

along with

$\operatorname{def}=18.075^{\circ}, a=1.79, \quad b=375.96, \quad c=-0.674$.

Declination triples are: $1640.5,6,5.73 ; 1814.5,-18.5$, $-17.75 ; 1815.5,-18.75,-17.71 ; 1836.0,-16.05,-16.5$; $1871.0,-12.28,-13.36 ; 1875.4,-11.26,-12.83 ; 1881.9$, $-10.73,-12.0 ; 1890.5,-10.21,-10.86 ; 1892,-10.08$, $-10.65 ; 1923.9,-6.37,-5.86$.

Dip triples are: $1836.0,57.26,57.20 ; 1871.0,54.5,54.7$; 1875.4, 54.3, 54.45; 1881.9, 54.13, 54.14; 1890.5, 53.98, $53.80 ; 1890.0,53.95,53.75 ; 1923.9,53.23,53.32$.

The anticlockwise loop of dip vs. declination at Palermo is shown in Fig. 13. The loop given by Strangway (1970), shown clockwise, appears to be irreconcilable with the data. 


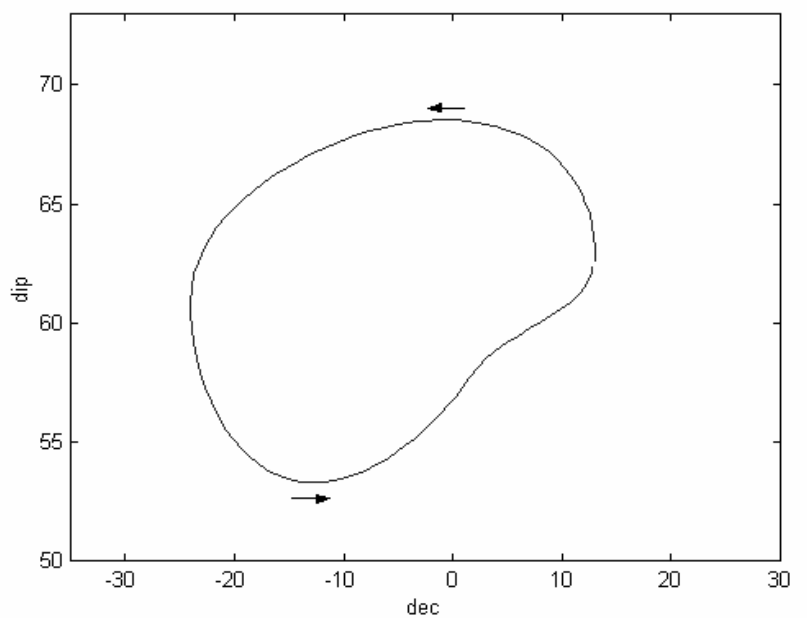

Fig. 13. Dip vs declination at Palermo.

A bonus on the Palermo Internet site is the availability of values for the horizontal component of the Earth's magnetic field,

$H=\sqrt{X^{2}+Y^{2}}$

Choosing the scaling factor, $g$, to give the same mean value for observations and computations yields

$g=20.49$

and the following triples: 1836.0, 24079, 24585; 1871.0, 24230, 24654; 1875.4, 24901, 24674; 1890.5, 25099, 24765; 1890.2, 25140, 24776; 1923.9, 25319, 25135.

\section{The problem of sunspots}

The model developed here can account for the major features of sunspot activity. In this case, the role of the mantle is taken by the photosphere (Priest, 1987) and secular variation occurs over a 22 year period. We suggest that the current $i_{d}$ is broken into filaments by bubbles rising through the convection zone and that these, on breaking free of the surface, are encircled by tubes of flux. If they settle back onto the Sun's surface, still carrying current, the flux drives out the surrounding plasma, giving rise to pairs of adjacent sunspots. When $i_{d}$ is positive, the right-hand member of a pair in the Northern hemisphere possesses North magnetic polarity and the left-hand member South magnetic polarity. This pattern is reversed during the following half cycle, which takes 11 years. Since $i_{d}$ is directed oppositely in the Southern hemisphere, events there mirror those in the North, on a probabilistic basis, but with reversed magnetic polarities. The general optical intensity of sunspot activity must reflect the product of the magnitude of $i_{d}$ with a measure of sub-surface turbulence. A very simple application of Ampere's Circuital Theorem indicates that the flux density at the centre of each

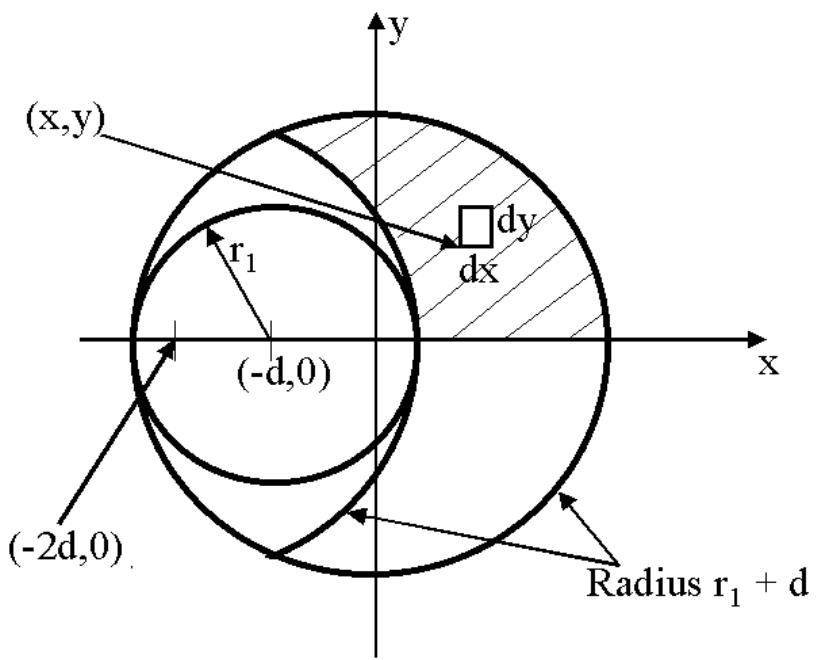

Fig. 14. Calculation of gravitational force on solid core.

spot of an adjacent pair increases monotonically with observed linear dimensions, as is inferred spectroscopically, using the Zeeman effect.

\section{Offset of the magnetic axis from the spin axis: some preliminary thoughts}

A globe, on which the Earth's magnetic poles are marked, shows that the magnetic axis is derived from the spin axis by translating the latter by approximately $10^{3} \mathrm{~km}$ towards the Pacific Ocean, out of the plane of the $120^{\circ}$ East $-60^{\circ}$ West circle of latitude. It is then turned clockwise by $11.5^{\circ}$, as viewed from the Pacific. It seems natural to assume that this reflects an offset and rotation from the geographic axis of the spin axis of the inner core. The offset would not need to be, for eample $10^{3} \mathrm{~km}$, since the magnetic poles are those points at which dip is $\pm 90^{\circ}$, and flux line curvature could greatly amplify the core offset in projecting it onto the Earth's surface.

Assuming that the solid core is in orbit, at a distance $d$ out from the spin axis, we have calculated four central forces, just to initiate thinking about this possibility.

\subsection{Gravitational force (inwards)}

The basis for calculation of this is shown in Fig. 14. The solid core is assumed to be a ball of radius $r_{1}$, having uniform density $\rho_{s}$, and the fluid core with uniform density $\rho_{f}$. We invoke the well-known result that a point mass within a uniform spherical shell experiences zero gravitational force. It is then readily appreciated that the gravitational restoring force on the solid core is due entirely to the fluid contained within the volume of revolution formed from the crosshatched cres- 


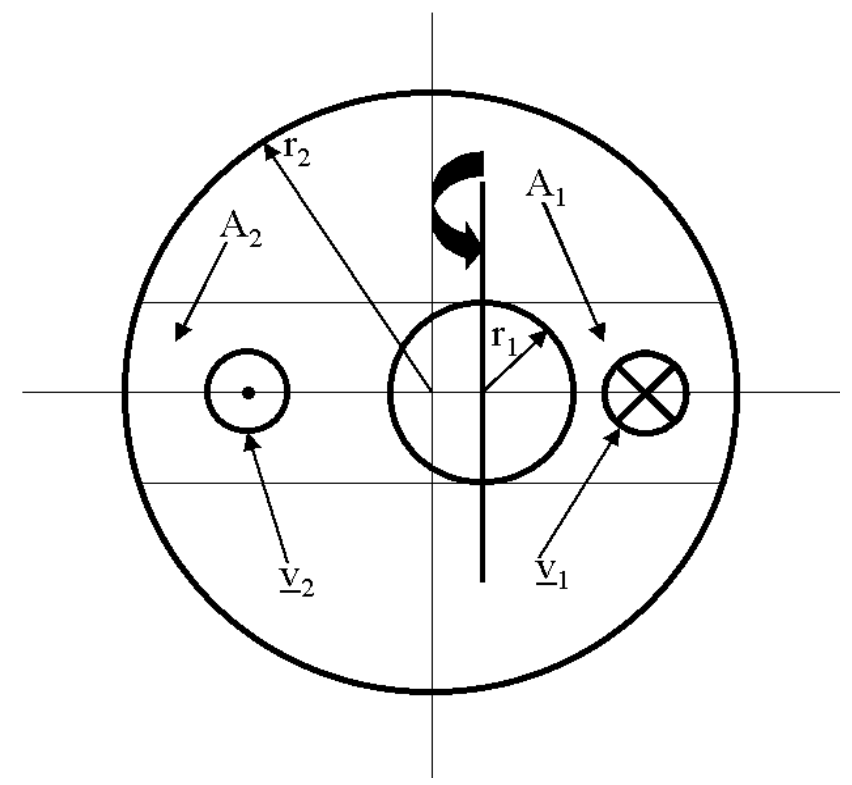

Fig. 15. Estimation of outward Magnus force on solid core.

cent. This force is given by the integral

$F_{g}=\frac{8 \pi^{2}}{3} G \rho_{s} r_{1}^{3} \rho_{f} \iint \frac{y(x+d)}{\sqrt{\left((x+d)^{2}+y^{2}\right)^{3}}} d x d y$

with appropriate limits. $G=6.673 \times 10^{-11} \mathrm{~m}^{3} \mathrm{~kg}^{-1} \mathrm{~s}^{-1}$ is the universal constant of gravitation. We have been able to evaluate this integral as

$F_{g}=\frac{16 \pi^{2}}{9} G \rho_{f} \rho_{s} r_{1}^{3} d$

\subsection{Buoyancy force (outwards)}

Replacing the solid core by a sphere of fluid, the gravity force on it would be given by Eq. (68), with $\rho_{s}$ replaced by $\rho_{f}$. This inward gravity force would be balanced by an outward buoyancy force, which would be unchanged if the fluid were again replaced by solid. Thus, the outward buoyancy force on the solid core must be given by the expression

$F_{b}=\frac{16 \pi^{2}}{9} G \rho_{f}^{2} r_{1}^{3} d$

\subsection{Centripetal Force (outwards)}

If the solid core were twirling about the spin axis at $\Omega$ radians per second (where $\Omega=7.27 \times 10^{-5} \mathrm{rad} \mathrm{s}^{-1}$, equivalent to one revolution per day), the centripetal force on it would be given by

$F_{c}=\frac{4 \pi}{3} \rho_{s} r_{1}^{3} d \Omega^{2}$

All three forces considered, so far, are linear in $d$. Taking $\rho_{f}=1.13 \times 10^{4}, \rho_{s}=1.34 \times 10^{4} \mathrm{~kg} \mathrm{~m}^{-3}$, and using $r_{1}=$
$1.25 \times 10^{6} \mathrm{~m}$, these combine into a single inward resultant

$F_{i}=F_{g}-F_{b}-F_{c}=5.368 \times 10^{16} d$

\subsection{Magnus Force (outwards)}

In 1925, Anton Flettner sailed his ship, the Baden-Baden, from Hamburg to New York, using the Magnus Force, a lift force experienced by vertical rotating cylinders in a horizontal wind stream (Wilkins, 1928). As with all lift, this is a consequence of the trade-off between pressure energy and kinetic energy along a streamline, as described by Bernoulli's equation. We attempt below to establish an admittedly, very crude way of estimating the Magnus force on the spinning solid core of the Earth, offset by $d$ from the centre of the Earth. The basis for the method is shown in Fig. 15.

For $d=0$, crosshatched areas are equal and have the value

$A=r_{1} \sqrt{r_{2}^{2}-r_{1}^{2}}+r_{2}^{2} \sin ^{-1}\left(r_{1} / r_{2}\right)-\frac{\pi}{2} r_{1}^{2}$

Under the condition shown, the right-hand area contracts to

$A_{1}=A-2 r_{1} d$

while the left-hand area expands to

$A_{2}=A+2 r_{1} d$

Considering only the fluid circulating through $A_{1}$ and $A_{2}$, with mean velocities $\left\langle v_{1}\right\rangle$ and $\left\langle v_{2}\right\rangle$, respectively, conservation of volume flow rate gives

$A_{1}\left\langle v_{1}\right\rangle=A_{2}\left\langle v_{2}\right\rangle$

This implies that there exists a function $g(d)$, such that

$\left\langle v_{1}\right\rangle=g(d)\left(A+2 r_{1} d\right)$
$\left\langle v_{2}\right\rangle=g(d)\left(A-2 r_{1} d\right)$

We are not in a position to derive $g(d)$ from first principles, but it is clear that it should have the following limiting values:

$g(0)=\frac{f r_{1} \omega}{A}$

$g\left(r_{2}-r_{1}\right)=0$

where $f$ is a positive fraction. The simple function

$g(d)=\frac{f r_{1} \omega}{A\left(r_{2}-r_{1}\right)}\left(r_{2}-r_{1}-d\right)$

is proposed for the present.

It is also suggested that $\left\langle v_{1}\right\rangle$ and $\left\langle v_{1}\right\rangle$ are obtained on the same streamline, so that Bernoulli's equation may be invoked to give the pressure difference

$p_{2}-p_{1}=0.5 \rho_{f}\left(\left\langle v_{1}\right\rangle^{2}-\left\langle v_{2}\right\rangle^{2}\right)$

The net outward Magnus force is now estimated as $F_{m}=$ $\pi r_{1}^{2}\left(p_{2}-p_{1}\right)$, i.e.,

$F_{m}=4 \pi r_{1}^{5} \rho_{f} f^{2} \omega^{2}\left(1-\frac{d}{r_{2}-r_{1}}\right)^{2} \frac{d}{A}$ 
It is interesting to note that $F_{m}=0$ at $d=0$ and $d=$ $\left(r_{2}-r_{1}\right)$, and that it has a positive peak at

$d=\frac{r_{2}-r_{1}}{3}=7.17 \times 10^{5} \mathrm{~m}$

The initial slope of the graph of $F_{m}$ vs. $d$ is $4 \pi r_{1}^{5} \rho_{f}$. $f^{2} \omega^{2} / A$, and the slope decreases monotonically with $d$ in the relevant range. If the initial slope were greater than that of $F_{i}$ vs. $d$, it would certainly denote a stable offset from the Earth's spin axis. However, the estimate $\omega=$ $8.3 \times 10^{-11} \mathrm{rad} \mathrm{s}^{-1}$ (Vidale et al., 2000) gives

$\left.\frac{d F_{m}}{d d}\right|_{0}=2.09 \times 10^{2} f^{2}$

This is so insignificant compared to the slope of the $F_{i}$ vs. $d$ line that if these were the only forces involved, and if the assumptions underlying them are reasonable, the position $d=0$ would be one of stable equilibrium. There are, however, other forces at play. Pinch effects, due to interaction of $i_{a}$ and $i_{d}$ with their magnetic fields, give outward forces for $d \neq 0$, as do electrostatic forces. There may also be more subtle aspects to the forces already considered, due to variation of fluid density with depth. That could have a dramatic effect on the difference between gravity and buoyancy forces. A comprehensive invetsigation of this problem is planned.

The author has been struck by the fact that a globe, observed from the Pacific side of the great circle, defined by the $120^{\circ}$ East $-60^{\circ}$ West lines of latitude, presents a hemisphere covered almost completely by water. Could this indicate that the solid core is, indeed, displaced to that side, and that resulting out-of-balance forces were responsible, at least in part, for driving continental drift to the other side, to maintain the overall centre of gravity on the axis of spin?

\section{Discussion}

Since this is the first exposition of a fairly radical theory, there are many ramifications still to be explored. One relates to the choice of $x_{3}$. The scaling relation between $x_{3}$ and $\omega$ is given in the third member of Eq. (21), with the physical parameters involved, approximated by Eqs. (53), (55) and (56). Vidale et al. (2000) suggest the small positive value $\omega \approx 8.3 \times 10^{-11}$ radians per second. This indicates a positive Hall Coefficient.

The numerical value of $x_{3}$ is not very critical, except that it must be in the range $x_{3}^{2}>1$ for the stable pseudo equilibrium point $P_{2}$ to exist, and must be chosen to give a lightly damped spiral, if indeed time-varying declination continues to be significant between reversals. Parameters $x_{3}$ and $\eta$ determine the shape and damping ratio of the spiral about $P_{2}$ in the $\left(x_{1}, x_{2}\right)$ plane. The damping ratio for linearised motion is given by the expression

$\zeta=\frac{1}{2 \eta \sqrt{f\left(x_{3}\right)}}$ with

$f\left(x_{3}\right)=2 \sqrt{x_{3}^{2}-1}\left(\left|x_{3}\right|+\sqrt{x_{3}^{2}-1}\right)$

The values $\left|x_{3}\right|=600$ and $\eta=1$ give $\zeta=.0004$, so that the spiral is lightly damped and the separatrix passes very close to the origin. This facilitates self-excitation by Johnson/Nyquist noise in $R_{a}$ and $R_{d}$. Choosing any higher value of $\left|x_{3}\right|$ decreases the value of $\zeta$, thus leading to easier selfexcitation and a greater number of subsequent significant oscillations.

It is a straightforward matter to deduce from Eqs. (83) and (84) that the spiral exists $\left(P_{2}\right.$ is a stable focus rather than a stable node) in the range

$\left|x_{3}\right|>1+\frac{1}{16 \eta^{2}\left(1+4 \eta^{2}\right)}$

For the value $\eta=1$, this gives $\left|x_{3}\right|>1.0125$.

Differential rotation in the Sun is significantly more pronounced than on Earth, giving a much smaller value of $\zeta$.

\section{Appendix A Peter Barlow's experiment}

There was an experiment performed 170 years ago, which relates to the basic geomagnetic viewpoint adopted here. It generated a current circulating in an annulus beneath the Earth's mantle, but since it lacked the counterpart of $i_{d}$, it could not shed any light on the time-variation of secular variations. It is hoped that it may be of historical interest to quote a description from Professor Peter Barlow. He published his account in 1831, the year before Michael Faraday discovered the law of electromagnetic induction. The following extract from Barlow's paper in Phil. Trans. Roy. Soc. is taken from pp. 268-271 of "A Treatise on Magnetism," by Sir David Brewster, Adam and Charles Black, North Bridge, Edinburgh, 1838.

"I procured," says he, "a wooden globe sixteen inches in diameter, which was made hollow for the purpose of reducing its weight; and, while still in the lathe, grooves were cut to represent an equator, and parallels of latitude at every 4 $1 / 2^{\circ}$ each way from the equator to the poles; and lastly, a groove of the same breadth, but of double the depth, was cut like a meridian, from pole to pole, half round. These grooves were for the purpose of laying in the wire, which was effected thus: The middle of a copper wire, nearly ninety feet long, and one tenth of an inch in diameter, was applied to the equatorial groove, so as to meet in the transverse meridian; it was then made to pass around this parallel, returned again along the meridian to the next parallel; then passed round this again; and so on, till the wire was thus led in continuation from pole to pole."

"The length of wire still remaining at each pole was bound with varnished silk to prevent contact, and then returned from each pole along the meridian groove to the equator. At this point, each wire being fastened down with small staples, the wires for the remaining five feet were bound together to near 
their common extremity, where they opened to form two points for connecting the poles of a powerful galvanic battery."

"When this connection was made, the wire became of course an electric conductor, and the whole surface of the globe was put into a state of transient magnetic induction, and consequently, agreeable to the laws of action above described, a neutralised needle freely suspended above such a globe would arrange itself in a plane passing from pole to pole through the centre, and take different angles of inclination, according to its situation between the equator and either pole."

"In order to render the experiment more strongly representative of the actual state of the Earth, the globe, in the state above described, was covered by the gores of a common globe, which were laid on so to bring the poles of this wire arrangement into the situation of the Earth's magnetic poles, according to the best observations we have for this determination. I therefore placed them in latitude $72^{\circ}$ north and $72^{\circ}$ south, and on the meridian corresponding with longitude $76^{\circ}$ west, by which means the magnetic and true equators cut one another in about $14^{\circ}$ east, and $166^{\circ}$ west longitude."

"The globe being thus completed, a delicate needle must be suspended above it, neutralised from the effect of the Earth's magnetism, according to the principle I employed in my observations of the daily variation, and described in the Philosophical Transactions for 1823; by which means it will become entirely under the superficial galvanic arrangement just described. Conceive now the globe to be placed so as to bring London into the zenith, then the two ends of the conducting wire being connected with the poles of a powerful battery, it will be seen immediately that the needle, which was before indifferent to any direction, will have its north end depressed about $70^{\circ}$, as nearly as the eye can judge, which is the actual dip in London; it will also be directed towards the magnetic poles of the globe, thereby also shewing a variation of about $24^{\circ}$ to $25^{\circ}$ to west, as is also the case in London. If we now turn the globe around on its support, so as to bring into the zenith places equally distant with England from the magnetic pole, we shall find the dip remains the same; but the variation will continually change, becoming first zero, and then gradually increasing to the eastward, as happens on the Earth. If, again, we turn the globe so as to make the pole approach the zenith, the dip will increase till at the pole itself the needle will become perfectly vertical. Making now this pole recede, the dip will decrease, till at the equator it vanishes, the needle becoming horizontal. Continuing the motion, and approaching the south pole, the south end of the needle will be found to dip, increasing continually from the equator to the pole, where it again becomes vertical, but reversed as regards its verticality at the north pole."

Barlow felt that he had "proved the existence of a force competent to produce all the phenomena, without the aid of any body usually called magnetic;" yet he acknowledged that "we have no idea of how such a system of currents can have existence on the Earth, because, to produce them, we have been obliged to employ a particular arrangement of metals, acids, and conductors." Michael Faraday was just about to change all that, and the Hall effect, discovered by Edwin Hall in 1879 , completed the prerequisites for the present study.

Acknowledgements. The author is extremely grateful to Professor Raymond Hide for introducing him to the problem of the Earth's magnetic field in 1998, and for supplying him with many papers and references. He also wishes to thank his friend, Professor Denis O'Sullivan of the Dublin Institute for Advanced Studies, for bringing the phenomenon of sunspot activity to his attention; and another friend, Brian Cogan, for helpful discussions and papers. He is deeply indebted to the National University of Ireland, Dublin, the National Rehabilitation Hospital, Dún Laoghaire, and the Electricity Supply Board (Ireland) for supporting his researches in many fields over many years. He wishes particularly to thank his students, Tomás Ward, David Burke, Ted Burke, Michael Wren, Thomas McHugh and Mary Doorly for generous help with references, computations and diagrams, and another student in his Department, Garret Brady, for checking the electromagnetic calculations. Thanks are also offered to Professor Edward Spooner of the University of Durham, the late Professor Oldrich Benda of the Slovak Technical University, Bratislava, and Professor Vladimir Kuznetsov of the Institute of Geophysics, Novosibirsk for reading the paper and making constructive criticisms.

\section{References}

de Paor, A. M., Nonlinear quenching of current fluctuations in a self-exciting homopolar dynamo, proved by feedback system theory, Nonlinear Processes in Geophysics, 5, 75-80, 1998a.

de Paor, A. M., In Haughton's Footsteps: Mathematical Insights into Bioengineering and Rehabilitation, Irish Journal of Medical Science, 167, 170-180, 1998b.

Hide, R., The nonlinear differential equations governing a hierarchy of self-exciting coupled Faraday-disk homopolar dynamos, Physics of the Earth and Planetary Interiors, 103, 281-291, 1997.

Hide, R., Skeldon, A. C., and Acheson, D. J., A study of two novel self-exciting single-disk homopolar dynamos: theory, Proc. Roy. Soc. Lond., 452, 1369-1395, 1998.

Hide, R., Nonlinear quenching of current fluctuations in a selfexciting homopolar dynamo, Nonlinear Processes in Geophysics, 4, 201-205, 1998.

Inglis, D. R., Theories of the Earth's Magnetism, Reviews of Modern Physics, 27, 212-248, 1955.

Larmor, J., How could a rotating body such as the Sun become a magnet?, Rep. Brit. Assoc. Adv. Sci., 159-160, 1919.

Lee, E. W., Magnetism, Penguin Books, Harmondsworth, 1963.

Lorenz, E. N., Deterministic nonperiodic flow, J. Atmos. Phys., 20, 130-141, 1963.

Lyapunov, A. M., The general problem of the stability of motion, Taylor and Francis, London, 1992. (Centenary translation by A. T. Fuller of the doctoral thesis submitted to the University of Kharkov, Russia, 1892.)

Malin, S. R. C. and Bullard, E., The direction of the Earth's magnetic field at London, Phil. Trans. Roy. Soc., A299, 357-423 (with two pullouts), 1981.

Moroz, I. M., Hide, R., and Soward, A. M., On self-exciting coupled Faraday-disk homopolar dynamos driving series motors, Physica D, 117, 128-144, 1998.

Ohanian, H. C., Classical Electrodynamics, Allyn and Bacon, Boston, 1988. 
Priest, E. R., Solar Magneto-Hydrodynamics, Reidel, Dordrecht, 1987.

Rikitake, T., Oscillations of a system of disk dynamos, Proc. Camb. Phil. Soc., 54, 89-105, 1958.

Shockley, W., Electrons and Holes in Semiconductors, Van Nostrand, Princeton, NJ, 1950.

Song, X. and Richards, P. G., Seismological evidence for differential rotation of the Earth's inner core, Nature, 382, 221-224, 1996.

Starling, S. G., Electricity and Magnetism for Degree Students, Longmans, Green \& Co., London, 1941.
Strangway, D., History of the Earth's Magnetic Field, McGrawHill, New York, 1970.

Turcotte, D. L., Fractals and Chaos in Geology and Geophysics, Cambridge University Press, Cambridge, 1992.

Van der Ziel, A., Solid State Physical Electronics, Prentice Hall, Englewood Cliffs, NJ, 1957.

Vidale, J. E., Dodge, D. A., and Earle, P. A., Slow differential rotation of the Earth's inner core indicated by temporal changes in scattering, Nature, 405, 445-448, 2000.

Wilkins, H. T., Marvels of modern mechanics: the mastery of land, sea and air, T. Fisher Unwin, London, 1928 (2nd edition). 